under the industrial conditions with a flux is still auxeticfree. It is proposed that a suitable substitute be tried for the flux, or it may be found more practicable to treat the palm oil or flux, or both, by reagents in order to oxidise the auxetics or to fix them by the Sorenson reaction. Further, it may be advisable to separate the stages of the process of tinning by suitable covering of the various parts of the process or by isolating the workmen.

Owing to difficulties which have occurred in the past, the foregoing suggestions and practical measures are hereby published in accordance with the desire of Mr. J. H. McFadden, the patron of this Research Fund, already communicated to H.M. Government, that no improvement in any manufacturing process arising out of work done at this Research Foundation shall be exploited commercially.

\section{A CASE OF VOLVULUS OF THE SMALL INTESTINE COMPLICATING GENERAL PERITONITIS ; RECOVERY.}

By O. Willett oUnNington, M.B., B.C. Cantab., CLINICAI ASSISTANT (DISEASES OF WOMEN), GREAT MORTHERN CENTRAL HOSPITAL.

VoLVULUS of the small intestine is a rare surgical event, and recovery from it is still more so. Occurring in a patient who is already suffering from general peritonitis, the combination terminating successfully must be reckoned as something of a "curiosity."

The clinical history of the case is as follows. On April 17th the patient, a thin, somewhat delicate-looking woman, aged 28 years, was seized with symptoms of acute appendioitis. She gave a history of frequent previous attacks, all of a slight nature. The physical signs were localised; the general symptoms were not very severe. The leucocytosis was 32,000 . The same day Mr. G. E. Gask removed the appendix, the tip of which was gangrenous and perforated. The region was wiped dry and a tube inserted in the usual way. The temperature and pulse fell, and for some days the patient's condition was satisfactory. On the 21st, however, slight abdominal distension was noticed. The next day this rapidly increased, the temperature rose to $101^{\circ} \mathrm{F}$. and the leucocytosis to 42,000 . The patient vomited several times. Accordingly, that evening the abdomen was opened again. The original wound was clean and well shut off by adhesions from the general peritoneal cavity. When the adhesions were broken down a secondary abscess was discovered and a large quantity of stinking pus was evacuated. A second opening was made above the pubes, and one drainage tube passed into Douglas's pouch and a second from the first to the second wound under the abdominal wall. The patient was returned to bed in Fowler's position, and continuous rectal salines were administered. Cultures of the pus showed bacillus coli and streptococcus brevis, and an autogenous double vaccine was prepared, the first dose being given the day following.

The patient's condition was grave, and was further imperilled by a smart secondary hæmorrhage from the fresh incision, producing temporary blanching and collapse. Both wounds became gangrenous and exceedingly offensive, and for some days the condition of general toxæmia was profound. Indeed, on the 25th she appeared to be sinking, being unable to retain any fluids by mouth or rectum. She rallied, however, the next day and began to emerge from the toxæmia, an improvement due in part, no doubt, to the effects of the vaccine, which was being given on alternate days in doses of $5,000,000$ of each organism. By May 4th she seemed to be getting " round the corner." That night, however, she complained of colicky pain and she vomited. The symptoms increased and the vomit became fæculent. At first flatus was passed with the help of enemata, but by the afternoon (May 5th) it was evident that complete obstruction had occurred.

The abdomen was opened for the third time by Mr. Gask, in the middle line through the umbilicus. As the two former wounds were still suppurating freely, the difficulty of preventing fresh infection was considerable, inasmuch as the coils of small intestine, greatly distended, had to be lifted out of the abdominal cavity. Eventually a volvulus of the small intestine was made manifest. It appeared to have arisen round an adhesion deep in the pelvic cavity.
A loop of small intestine was completely twisted upon itself and fixed by recent adhesions. These were gently separated and the loop cautiously untwisted. These manipu. lations produced a good deal of shock. The distended loop was rapidly sewn into the wound and a small puncture then made into it, from which escaped much gas and fluid. While recovering from the anæsthetic the patient vomited quantities of fæculent fluid. Two days later the fistula was discharging bile, suggesting that the opening was high up in the small intestine. All distension subsided and the bowels were opened daily by enemata, but the patient suffered much from the constant discharge from the fistula and the excoriation of the skin produced by it. On May 14th the edges of the puncture were drawn together by a suture, but as this cut out, on the 16th the abdomen was opened for the fourth time and the fistula closed in the usual way with a double row of Lembert sutures and the coil of intestine freed from the wound and dropped back into the abdominal cavity. The abdominal wound was then closed, leaving the skin layer gaping on account of its septic condition. The patient made a good recovery, and by June 15th was sufficiently convalescent to go for a drive. On June 17 th she was again seized with symptoms of acute obstruction; and for the fifth time Mr. Gask opened the abdomen and found that the small intestine was obstructed by an adhesion, which was easily separated. From this date recovery was speedy and uninterrupted, and the patient is now in excellent health.

To ease is, I think, remarkable for several reasons. The rarity of volvulus of the small intestine and recovery from it under such disadvantageous circumstances (for at the third operation the presence of generalised peritonitis was conspicuous to the eye); the singular fate of a patient to have to experience five abdominal sections in such a short time, and her strange good fortune to have survived these ordeals, unite to make a remarkable combination. The fortitude with which the patient bore her vicissitudes had much to do with the happy result.

My best thanks are due to Mr. Gask as surgeon and to Mr. W. Stanley Rooke as anæsthetist at each operation. I have also to thank Mr. E. C. Cunnington for kindly collecting from the records at St. Bartholomew's Hospital statistics of all cases of volvulus admitted to that institution in nine years, which I add for the sake of comparison. Sixteen cases: 4 of large intestine, 11 of small intestine, and 1 unidentified. Of those identified, 2 of the large intestine recovered and 1 of the small intestine. The remainder were fatal.

Finchley, N.

\section{THE PREVALENCE OF CHRONIC GASTRIC DISEASE IN SPAIN :}

\section{NOTES ON MEDICAL AND SURGICAL TREATMENT.}

BY W. A. MACKAY, M.D. EDIN., F.R.C.S. EDIN., AND

IAN MACDONALD, M.D. PARIS \& EDIN.

IN Southern Spain the line between medicine and surgery is ill-defined. The beaten track of the specialist is stil untraced; this record, therefore, of gastro-enterostomy bas been made in a practice both medical and surgical. In the selection of cases for operation most types of dyspepsia have passed through our hands, and though in many cases of chronic gastric and duodenal ulcer the brilliant results of surgery are convincingly evident, in others we recognise the merits of careful medical treatment by daily enforcing it before advising operation.

Chronic disease of the stomach is very common here, and is most inefficiently treated ; relapses are frequent. Patients suffering pain and vomiting are rarely ordered rest in bed, that essential of successful treatment. Frequently such patients come anticipating operation because organised treatment has been totally wanting. They find the $\mathbf{s}^{2}$ symptoms disappear rapidly under rest and appropriate régime.

Ulcers in many cases begin most insidiously withont history of acute attacks. In this country among all classes and both sexes, but more so among males, there is a great irregularity of meals. Much raw indigestible food is 